\title{
Exploring the Relation between EMG Sampling Frequency and Hand Motion Recognition Accuracy
}

\author{
HongFeng Chen, Yue Zhang, Zhuo Zhang \\ College of Computer Science and Technology \\ Zhejiang University of Technology \\ Hangzhou, China PR \\ chenhf1026@gmail.com
}

\author{
Yinfeng Fang \\ School of Computing \\ University of Portsmouth \\ Portsmouth, United Kingdom \\ yinfeng.fang@port.ac.uk
}

\author{
Honghai Liu \\ School of Mechanical Engineering \\ Shanghai Jiao Tong University \\ Shanghai, China \\ honghai.liu@sjtu.edu.cn
}

\begin{abstract}
Myoelectric control with surface EMG signal has achieved great success in clinics, but only limited to the control of 2-Degrees-of-freedom prosthesis. With the appearance of multiple-channel and high-density EMG system and the advances of pattern recognition technology, it becomes possible to control a multi-degree smart prosthesis using EMG signals. However, it requires high performance EMG systems with high sampling frequency, which impedes the popularity of EMG-based applications. This study aims to explore a way to reduce the cost of EMG system by investigating the effect of sampling rate on gesture recognition accuracy. Two groups of experiments on inner-group and cross-group were designed to evaluate the classification accuracy at different EMG sampling frequency. In comparison with the sampling frequency at $1 \mathrm{kHz}$, a lower sampling frequency at $400 \mathrm{~Hz}$ could achieve comparable accuracy, reduced by only $0.43 \%(\mathrm{KNN})$ and $0.83 \%$ (SVM) with the overall accuracy at $99.40 \%$ and $98.67 \%$, respectively. It implies that appropriate reduction of the sampling frequency can be a good choice to balance the cost and performance of a multiple channel EMG system for feature-based hand gesture classification.
\end{abstract}

Index Terms-Surface EMG, Sampling rate, Prosthesis, Hand Motion, Pattern Recognition

\section{INTRODUCTION}

The Electromyopgraphy (EMG) is a biomedical signal, which is acquired by the electrical response generated in muscles throughout its contraction symbolizing neuromuscular activities (contraction/relaxation) [1]. Currently, there are two ways or methods of recording EMG signal: needle/internal EMG and surface electrode. The former requires a fine wire electrodes to be injected close to the muscle, and thus few scholars [2] use it for research. The latter is an onedimensional time series signal from the surface of muscle through the electrode guide and recorded neuromuscular system activity, which can be used easily without requiring medical doubters.

The surface EMG signal can directly reflect the motion intention of the human body, this means a hybrid electrical signal generated from the organ that performs a physical movement. The changes of surface EMG signal are related to the number of exercise units participating in the activity, the mode of movement unit and the metabolic state. So it can reflect the neuromuscular activity to a certain extent, and be significant in the diagnosis of neuromuscular diseases in clinical medicine, ergonomic analysis of ergonomics in the field of ergonomics and other important practical value [3]-[8]. At present, the human-computer interaction system based on sEMG has been applied to a variety of new service robots, such as intelligent artificial limb [9]-[12], rehabilitation robot [13]-[15] and behavioral assistant robot [16], etc. The core technology of using sEMG to realize intelligent humancomputer interaction is to accurately identify the body's movement intention by sEMG signal [17]. sEMG signal has also attracted remarkable attention to be used as the input source for man-machine interfaces, such as gesture recognition based game control and institutive prosthesis manipulation. In addition, multi-functional prosthetic hands with EMG PRbased control (Comparing with traditional methods, it can extract more useful information from specified muscles, thus more intuitive and effective control for myoelectric prostheses will be provided [18].) have been appeared in the market recent years, which brings great help to disabilities.

Based on these applications, sEMG acquisition devices need to be used to capture, store and analyze sEMG signal. Besides, an analog to digital (A/D) converter is always required for signal digitalization, in which the sampling rate is an important part in the converter. Several authors have suggested that the highest frequency components of the EMG signal are around 400-500 Hz [19], [20]. To follow the Nyquists rate, a smapling rate at least, $800-1000 \mathrm{~Hz}$ is needed to avoid aliasing and other signal distortion [21].

Generally, it is able to contain most information by using the sampling rate that exceed above values in the system, whereas several authors propose higher sampling rates. Gitter, etc., suggest that the sampling frequency should be 3-10 times the maximum frequency content because of the slow Nyquist rate [22], and when the sampling rates is lower than 5 times the maximum frequency of the signal, it will limit the waveform and cause the error of turns and spike amplitudes for waveform patterns [23]. Nilsson, etc., also found that when sampled below 3 times using a generated sinusoidal waveform, it would generate marked decreases in signal amplitude [24]. Jeffrey, etc., explore the effects of sampling rate on the time and amplitude of surface EMG signals. And they drew a different conclusion: In typical kinesiological investigations, it is not necessary to oversampling sEMG above the Nyquist rate, and a $1 \mathrm{kHz}$ sampling rate can be considered the functional Nyquist 
rate. Besides, they do not advise to smooth and undersample the sEMG data [25].

Anyway, it is clear that insufficient sampling frequency can result in distortion of the sEMG signal, such as the frequency content and waveform shape. But in fact there is little concrete evidence to show how this adverse effects will affect the common measures in the sEMG records (for example the amplitude), and moreover affect the accuracy of the feature-based hand motion recognition. In the other word, it is necessary to find the effect of undersampling on the degree of motion recognition declines and the most fitted sampling rate to obtain a cost-effective system. Therefore, the purpose of this investigation was to explore the specific impact of undersampling rate on the accuracy of the motion recognition with the EMG signal and determine if undersampling is a viable option in the real system.

\section{MATERIAL AND METHODS}

\section{A. Subjects}

Five apparently healthy volunteers ( 1 female, 4 males, age: $26 \pm 5$, height: $171 \pm 6 \mathrm{~cm}$, mass: $64 \pm 6 \mathrm{~kg}$ ) were provided informed consent, and under the institutional guidelines, they participated in the entire experimental process. Besides, they had no previous neurological history or traumas to the upper limbs.

\section{B. Apparatus}

A multi-channel sEMG system (as seen in Fig. 1) for forear$\mathrm{m}$ sEMG signal acquisition was customized for data collection in the experiment [26], [27]. The device mainly consists of the following four parts: 16 bipolar EMG channels (either single or jointly measured), 5000 amplifying gain, $1 \mathrm{kHz}$ sampling frequency and 12-bit ADC resolution. The system also includes a band-pass filter with the cut-off frequency at approximately $20 \mathrm{~Hz}$ and $500 \mathrm{~Hz}$, respectively. In addition, in order to remove the noise in the power line, a $50 \mathrm{~Hz}$ nontrapping filter is integrated in this device.

As an important part of the capture sEMG signal system, electrodes are fixed on an elastic fabric sleeve, and the material of it is made from Nylon and Spandex. Besides, the distances between electrodes is related to arm sizes. As shown in Fig. 1 (b), zig electrode layout is used in this experiment, which has been proved to be able to enhance the accuracy of action recognition and better robustness, comparing with the traditional parallel electrode layout [28].

\section{Data Collection}

Subjects are required to remain in the seat and keep the upper arm stationary when collecting the EMG signal. In this experiment, each subject need three groups of tests and the interval of two test is half an hour, which keep the muscle at the same level of fatigue. As shown in Fig. 2, each test contains eight actions, and each channel of the signal will be collected. In the experiment, a trial means the procedure that the force of sustained hand motion changes from the lightest strength to the greatest strength, and then back to the lightest.

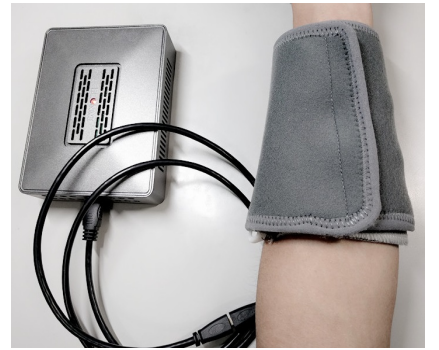

(a)

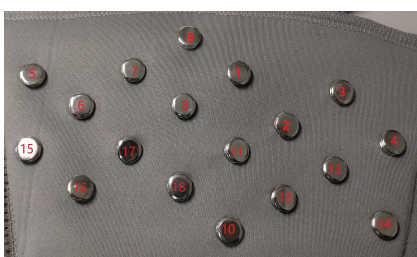

(b)
Fig. 1. This picture shows the device used in this experiment. (a) A muscle electrical apparatus comprising 16 channels, which can be used to gather single channel data or be matched with electrode sleeve for multi-channel data acquisition. (b) Electrode sleeve, which contains 18 electrodes altogether (The number 9 and 10 are two reference electrodes, and the remaining are 16 signal channels). It can be divided into two connected rings: 1-8 for one, and 11-18 for the other. This electrodes configuration was named Zig Configuration.

An experimental session means a subject wearing the electrode sleeve, continuously demonstrate the eight actions above. And each subject contains three sessions of data collection.

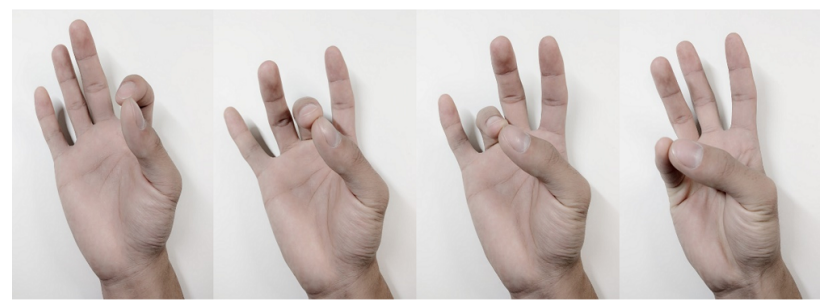

$(1)$

(2)

(3)

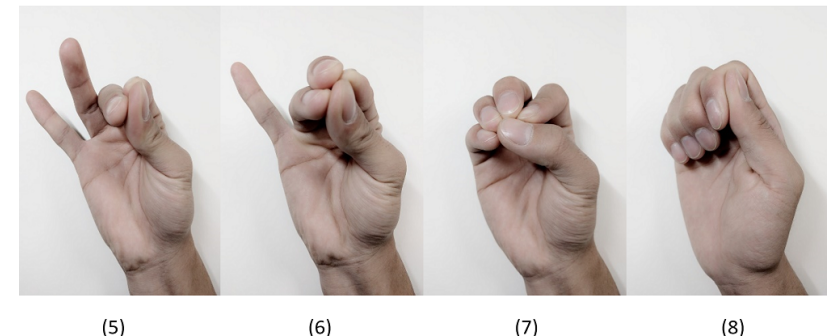

(5)

(6)

(7)

(8)

Fig. 2. Eight hand motions are used in this paper for hand motion recognition. 1-8 using thumb to touch index finger, middle finger, ring finger, little finger, two fingers, three fingers, four fingers, and the middle of index finger, respectively.

The experiment also includes a force sensor to detect the changes of power. During the test, each action was maintained for 100 seconds: the first 10 seconds, without effort; the 1120 s, increase the power gradually, and reach the peak at the 20 seconds; the 21-30s, reduce the power slowly, and in the 30 seconds to reach the minimum; the $31-40$ s, remain the gesture and keep force unchanged; the 41-50s, increase the power gradually, reach its peak at the 50 seconds; the 51-60s, reduce the power slowly, and reach the minimum at the 60 seconds; the 61-70s, remain the gesture and keep force unchanged; the $71-80$ s, increase the power gradually, and reach the peak at the 80 seconds; the 81-90s, reduce the power slowly, and reach the minimum at the 90 seconds; the $91-100$ s, keep gesture and power. 
It is not advisable to calibrate the electrodes and skin before the data signals were collected, because the bias of the electrodes will be generated more randomly in this mode, and it will enhance the diversity of EMG data [29]. In the process of wearing the electrode sleeve, participants must ensure that the reference electrodes stays on the upside of the forearm. After wearing the sleeve, it is suggested to wait for several minutes to reduce the skin to electrodes resistance naturally, which could enhance the effect of EMG signal acquisition.

\section{Signal Processing}

System response time and the final classification accuracy are closely related with the data segment [27], so the experiment extracts the characteristics of the data through the sliding window. Specifically, a $300 \mathrm{~ms}$ window and a $50 \mathrm{~ms}$ incremental window were used in the whole process. The former can provide enough information, while the latter can meet real-time requirements [30].

EMG features are extracted rather than using the raw EMG signal as the input. The experiment combines the autoregressive coefficient (AR) model [31] and time domain (TD) feature [32]. Among them, AR4 is chosen to produce four features per channel and the following three time domain features were selected to achieve satisfactory results: Root Mean Square (RMS), Waveform Length (WL), Mean Absolute Value (MAV) [26]. For each action, due to the instability of the EMG signal, only the steady-state signals were extracted. And in this experiment, the effective signals were included in the time period in which the force changed continuously, as shown in Fig. 3 (a), when the signal in these time periods: 11-30s; 4160s; 71-90s, the force is changing constantly: first increase and then decrease. Similarly, Fig. 3 (b) shows 4 paths in the 16 channels. Consequently, the sample size of a hand motion in one session is 9480 (395 samples in one trial, 3 trials for one motion and totally 8 motions). After the extraction of the features, three traditional classification methods were used in this experiment: K Nearest Neighbor (KNN), Support Vector Machine (SVM) and Linear Discriminant Analysis (LDA).

The experiment was divided into two parts: inner-group testing and cross-group testing. The former disrupted all the data and extracted $70 \%$ of it to be used as training data, and the remaining 30\% were used for testing. The specific experimental steps were as follows: First, re-extract the signal data in order to change the system sampling frequency, since we initially chose the sampling rate of $1 \mathrm{KHz}$ to collect the signal in the hardware device; Then, extract four features mentioned above from the segmented data signals, combine them to a new feature and carry on the operation of dimensionality reduction; Finally, three classification methods were used for gesture recognition. The experimental process of the latter part was similar to inner-group, the difference is that it chose two groups for training, and the rest for testing. All of the training and testing data above were collected by the same electrode sleeve. In this experiment, we selected 14 frequency values, starting from the largest $1 \mathrm{KHz}$ and descending. The initial descent step was 100 , when the frequency dropped to $100 \mathrm{~Hz}$,
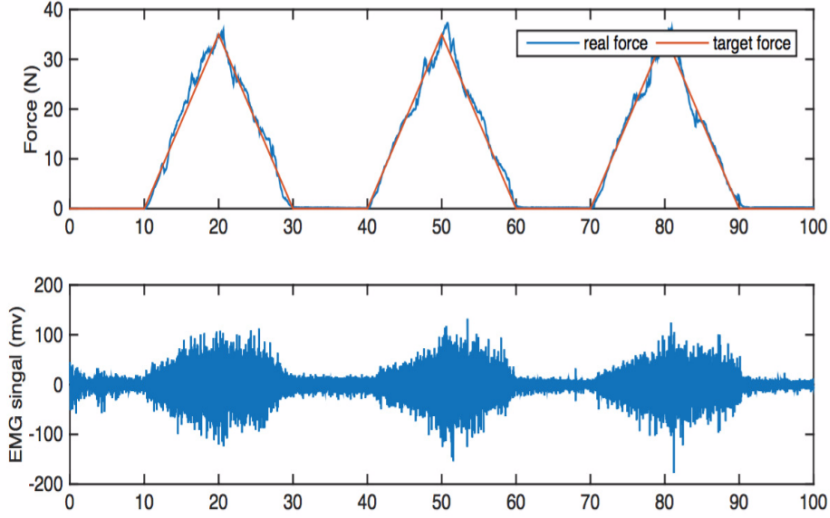

(a)
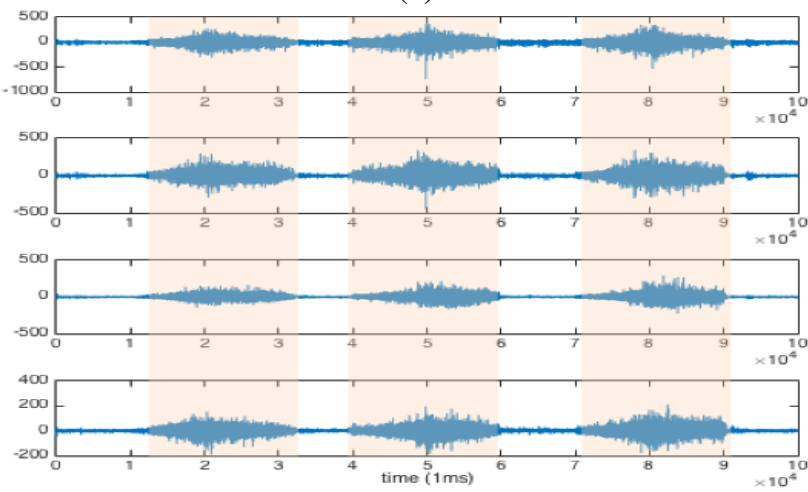

(b)

Fig. 3. This picture shows the EMG signal. (a) It shows the amplitude variations of the single channel EMG signal, as well as the change of the force, which reflects the whole collecting signal process. (b) Collecting data from 4 channels, which has the similar trend of amplitude variation.

the descent step became to 20 , and it can be observed from the following results that this selection method can reflect better performance.

\section{RESULTS AND DISCUSSION}

\section{A. Inner-group Testing}

The surface EMG signal is nonstationary, and it is easy to be interfered by external environment [33], therefore, in order to explore the effect of different sampling rates on gesture recognition, a data set that we randomly select one from the same session does not represent the final experimental result. We tested 5 subjects with all 15 sessions, using the cross validation method for each session, and finally obtaining the average value (In this experiment, we assume that the data sets in different sessions are independent for each other). The result can be seen in Fig. 4. Obviously, no matter which method was used, gesture recognition reduced with the decrease of sampling rate, and the use of LDA algorithm for this data set was worse than other two methods, where KNN can still achieve a good effect. This may because the amount of data collected in this experiment is approximately equal, the data latitude is high, and the sample assemblies to be sorted have a variety of phenomena of overlapping or crossing, while the 
KNN method mainly relies on the limited neighboring sample, rather than the method of distinguishing the class domain, so it has a better performance.

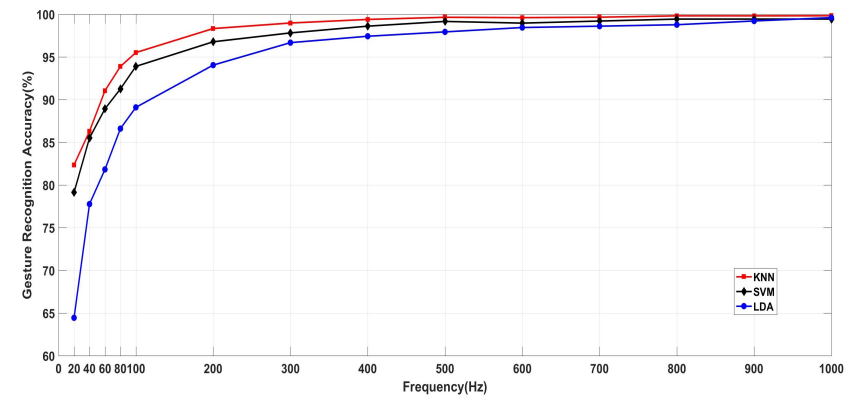

Fig. 4. The average classification rate with the sampling rate decreasing constantly by using KNN, SVM and LDA algorithm among one session.

Whichever method to be chosen, the $\mathrm{P}$ value can be used to analyze the trend of declining of recognition rate, which was calculate in the two-side single sample T-test (Using the KNN algorithm for classification). First, we can assume H0: when the sampling rate drops from $1 \mathrm{KHz}$ to $200 \mathrm{~Hz}$, the gesture recognition rate will drop significantly, more than $2 \%$. And the alternate assumption $\mathrm{Ha}$ is: when the sampling rate drops from $1 \mathrm{KHz}$ to $200 \mathrm{~Hz}$, the degree of declining in recognition rate is low, within $2 \%$. In this experiment, the data trained by the KNN classification algorithm generate randomly. Therefore, after 30 cycles we can get the corresponding 30 differences, that is, the amplitude of the recognition rate. At this time, we know the sample size: $n=30$, and according to the experimental results, we can calculate sample mean: $X=0.01697$, sample variance: $s^{2}=0.000014$, and the standard error for sample mean: $s(\bar{X})=\sqrt{s^{2} / n}=0.000683$. Then, we assume $\mathrm{HO}$ is true, while the sample mean is an estimate, which is only obtained from a random sample of the population (the sample is 30 cyclic random operations). We don't know whether this result is an "extreme" event, so we need to repeat the experiment above and find out a sample value for each one. Finally, it is easy to know that the calculated sample mean obeys a $t$ distribution with a freedom of 29 , that is,

$$
(\bar{X}-\mu) / s(\bar{X}) \sim t(29)
$$

So there is a corresponding $\mathrm{t}$ value $(\mathrm{t}=(0.01697-0.02) / 0.000683$ $=-4.4363$ ) under the $t$ distribution of 29 degrees of freedom, with the sample mean $X=0.01697$. And observe this $\mathrm{t}$ value throughout the whole distribution, we can calculate that points which are more extreme than this value account for 0.0001 of the entire distribution, that is, $P=0.0001$. It is much less than the significant level: $\alpha=0.05$. Thus, rejecting the assumption, and it can be concluded: Using the KNN classification algorithm to the experimental data, when the sampling rate drops from $1 \mathrm{KHz}$ to $200 \mathrm{~Hz}$, the degree of declining in recognition rate is low, within $2 \%$. Other cases are similar, which means the recognition rate is indeed decreasing, and the amplitude of reduction is small in the initial phase.
Next, considering the sensitivity of single feature to the sampling rate, and the result was carry out by using three traditional classification methods. As shown in Table 1, these three characteristics (RMS, WL, MAV) have a similar degree of sensitivity to the sampling rate: when the sampling rate is reduced slowly from $1 \mathrm{KHz}$, the recognition rate of the classification can be remain roughly constant or reduced at a very slow rate, and when the sampling rate is as low as a certain frequency, for example $200 \mathrm{~Hz}$, its recognition rate will be dropped sharply. In contrast, the characteristic AR4 is more sensitive to the sampling rate, which can be expressed in the following two points: 1) Using SVM classification algorithm, the recognition rate is lower than other three features, and at the same time, it drops significantly. 2) Using KNN or LDA classification algorithm, the recognition rate is higher than the other three characteristics at the early stage, and when the sampling rate is close to a certain frequency (for example $200 \mathrm{~Hz}$ ), the recognition rate will decline sharply, and the magnitude of the reduction is the largest.

\section{B. Cross-group Testing}

In order to reveal the specific relationship between sampling rate and gesture recognition rate, some exploration will be carried out between different sessions. Each subject contained three sessions and two of them were selected to produce a feature set used as the training data. In contrast, the remaining session was used as the testing data. The same classification methods were used for identification. 5 participants conducted the process above and the average values were obtained. The result can be seen in Fig. 5, when the sampling rate is in the frequency range of $200 \mathrm{~Hz}-1 \mathrm{KHz}$, the recognition rate of gesture is about $80 \%$, and it decreases rapidly when the sampling rate is further reduced. This trend is basically the same as the result of using single feature and feature combination.

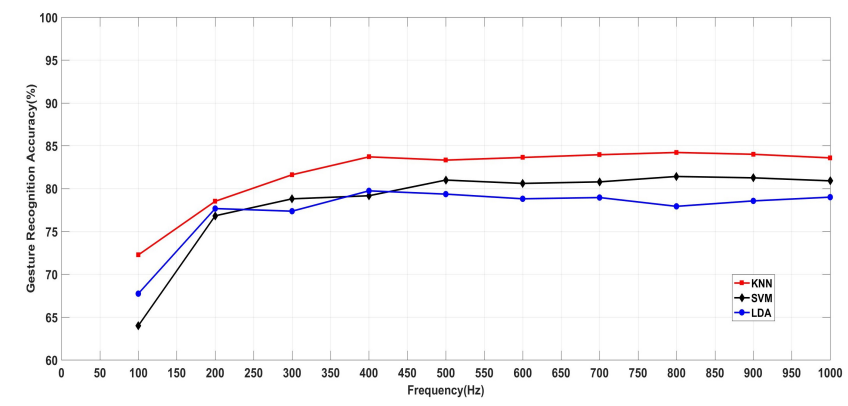

Fig. 5. The effect of the gesture recognition rate on the changing sampling rate between different sessions.

\section{Discussion}

The experimental results show that the trend of gesture recognition rate is similar in the test of inner-group and crossgroup with the gradual decrease of sampling rate, whether using the single feature or mixing feature, LDA, KNN or SVM algorithm. And it is not necessary to use the maximum signal 
frequency of twice times or even higher sampling rate. For example, the RMS eigenvalues at different sampling rates, we can see from the Fig. 6 (a) that the $500 \mathrm{~Hz}$ signals is quite similar to $1 \mathrm{KHz}$ signals, and the $200 \mathrm{~Hz}$ signals look normal, but the $100 \mathrm{~Hz}$ signals have a lot of difference comparing with three kind of signals mentioned above. This is mainly due to the reduction of sample size, when connecting adjacent points, the graphics will behave more "steep". And for a specific action (such as gesture one in Fig. 2), although the coverage of frequency value is basically within $0-500 \mathrm{~Hz}$, we can observe that the weight of the frequency component around $100 \mathrm{~Hz}$ is the largest from the Fig. 6 (b). So reducing the sampling rate in the initial phase, the collected signal can still contain the low frequency information. Then, applying it to gesture recognition, the loss of information may not as great as it might have been. However, when the frequency drops to a certain extent (as described earlier), it may even lose the information of this component, so the recognition rate will have a large decline. The specific results are as follows: when the sampling rate drops gradually from $1 \mathrm{KHz}$ to $200 \mathrm{~Hz}$, using three methods (KNN, SVM, LDA) for the EMG signal, the corresponding gesture recognition rate decreased from $99.82 \%$ to $98.32 \%, 99.44 \%$ to $96.77 \%, 99.6 \%$ to $94.04 \%$, respectively. And when the sampling rate dropped from $200 \mathrm{~Hz}$ to $100 \mathrm{~Hz}$, the recognition rate decreased rapidly, descending to $95.51 \%$, $93.89 \%$, and $89.29 \%$. It also can be observed from the above illustration that in the experimental process of decreasing sampling rate, KNN algorithm has a better classification effect.

\section{CONCLUSUION}

In this paper, 11 different frequencies, 4 kinds of EMG features, 3 classification algorithms were discussed for hand motion recognition. Three sets of experiments were designed to investigate the effect of sampling rate on the classification accuracy with surface EMG signals. The experiment shows that for the EMG signal data which the force changes constantly, LDA classification algorithm does not get a good result, while KNN algorithm can still maintain an ideal effect. And $1 \mathrm{KHz}$ sampling frequency with conventional EMG features (TD+AR4) for gesture classification accuracy is not necessary: when the sampling frequency was set to $400 \mathrm{~Hz}$, the recognition accuracy dropped by $0.43 \%$ for $\mathrm{KNN}$ and $0.83 \%$ for SVM, to $99.40 \%$ and $98.67 \%$, respectively. And when the frequency dropped down to $200 \mathrm{~Hz}, 1.5 \%$ to KNN. Therefore, the classification accuracy of surface EMG signals will not have a significant impact with the appropriate reduction of the sampling frequency, which has an important reference value in practical applications.

The higher the sampling rate, the more data were collected in unit time, more stringent requirements for hardware equipment. Such as a modular conversion (A/D) module, which requires a higher resolution, better dynamic characteristics and stronger conversion performance. Correspondingly, the cost can be increased greatly. Therefore, in the practical application of the equipment performance and accuracy without special
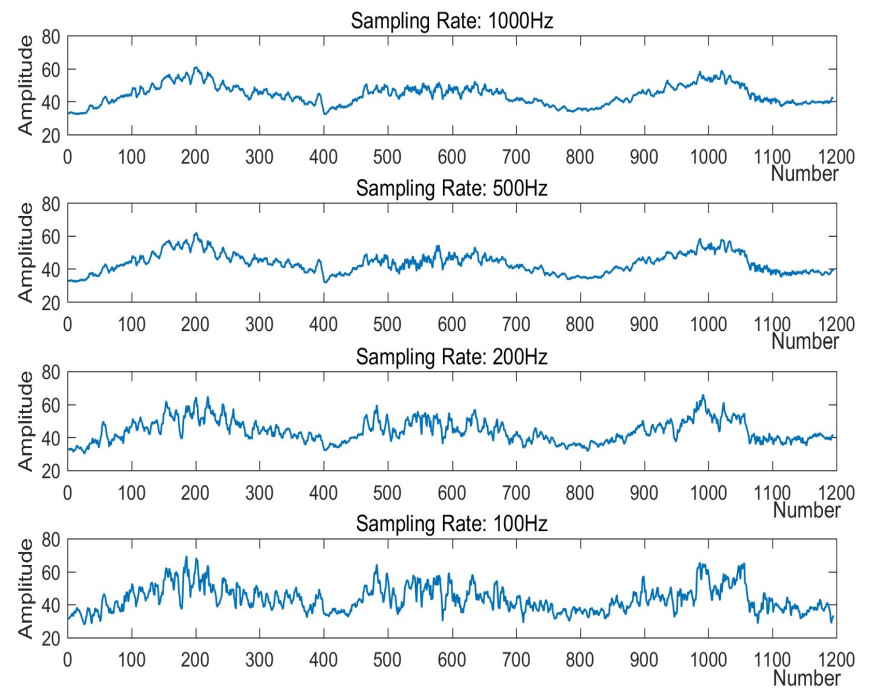

(a)

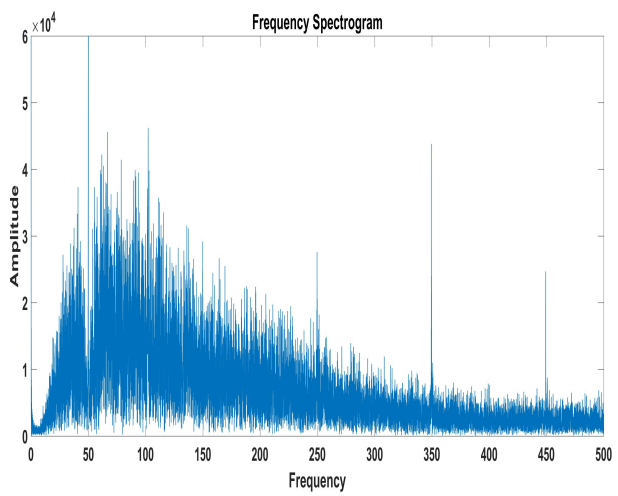

(b)

Fig. 6. (a) Representative trial from one subject sampled at $1 \mathrm{kHz}$ and resampled at $500 \mathrm{~Hz}, 250 \mathrm{~Hz}$ and $100 \mathrm{~Hz}$ (resampling at other rates not shown). (b) Spectrum diagram of a single action.

requirements, the appropriate reduction of the sampling rate will be a very desirable option.

\section{REFERENCES}

[1] S. A. Fattah, M. A. Iqbal, M. A. Jumana, and A. B. M. S. U. Doulah, "Identifying the motor neuron disease in emg signal using time and frequency domain features with comparison," Signal \& Image Processing, vol. 3, no. 2, pp. 99-114, 2012.

[2] C. Cipriani, J. L. Segil, J. A. Birdwell, and R. F. F. Weir, "Dexterous control of a prosthetic hand using fine-wire intramuscular electrodes in targeted extrinsic muscles," IEEE Transactions on Neural Systems \& Rehabilitation Engineering, vol. 22, no. 4, pp. 828-836, 2014.

[3] P. S. Lum, C. G. Burgar, and P. C. Shor, "Evidence for improved muscle activation patterns after retraining of reaching movements with the mime robotic system in subjects with post-stroke hemiparesis," IEEE Transactions on Neural Systems \& Rehabilitation Engineering A Publication of the IEEE Engineering in Medicine \& Biology Society, vol. 12, no. 2, pp. 186-194, 2004.

[4] P. S. Lum, C. G. Burgar, and P. C. Shor, "Use of the mime robotic system to retrain multi-joint reaching in post-stroke hemi-paresis: why some movement patterns work better than others," Engineering in Medicine and Biology Society, Proceedings of the International Conference of the IEEE, vol. 12, pp. 1475-1478, 2003. 
TABLE I

THE EFFECT OF THE GESTURE RECOGNITION RATE ON THE CHANGING SAMPLING RATE USING THE SINGLE FEATURE IN DIFFERENT CLASSIFICATION ALGORITHMS.

\begin{tabular}{|c|c|c|c|c|c|c|c|c|c|c|c|c|}
\hline Featrue & \multicolumn{3}{|c|}{ RMS } & \multicolumn{3}{c|}{ WL } & \multicolumn{3}{c|}{ MAV } & \multicolumn{2}{c|}{ AR4 } \\
\hline Mampling & \multirow{2}{*}{ KNN } & \multirow{2}{*}{ SVM } & LDA & \multirow{2}{*}{ KNN } & SVM & LDA & KNN & SVM & LDA & KNN & SVM & LDA \\
\hline $1000 \mathrm{~Hz}$ & 0.9969 & 0.9955 & 0.9172 & 0.9987 & 0.9945 & 0.9521 & 0.9963 & 0.9930 & 0.9045 & 0.9977 & 0.9910 & 0.9813 \\
\hline $800 \mathrm{~Hz}$ & 0.9932 & 0.9943 & 0.9107 & 0.9981 & 0.9938 & 0.9425 & 0.9960 & 0.9920 & 0.8965 & 0.9970 & 0.9742 & 0.9687 \\
\hline $600 \mathrm{~Hz}$ & 0.9961 & 0.9906 & 0.8964 & 0.9960 & 0.9886 & 0.9254 & 0.9951 & 0.9865 & 0.8811 & 0.9973 & 0.9479 & 0.9473 \\
\hline $500 \mathrm{~Hz}$ & 0.9960 & 0.9937 & 0.9047 & 0.9965 & 0.9915 & 0.9285 & 0.9952 & 0.9883 & 0.8899 & 0.9982 & 0.9386 & 0.9601 \\
\hline $400 \mathrm{~Hz}$ & 0.9955 & 0.9920 & 0.8967 & 0.9942 & 0.9863 & 0.9168 & 0.9946 & 0.9895 & 0.8785 & 0.9969 & 0.8909 & 0.9523 \\
\hline $300 \mathrm{~Hz}$ & 0.9921 & 0.9859 & 0.8677 & 0.9899 & 0.9759 & 0.8843 & 0.9916 & 0.9816 & 0.8586 & 0.9964 & 0.8661 & 0.9279 \\
\hline $200 \mathrm{~Hz}$ & 0.9827 & 0.9621 & 0.8374 & 0.9831 & 0.9659 & 0.8638 & 0.9785 & 0.9398 & 0.8162 & 0.9854 & 0.7830 & 0.8526 \\
\hline $100 \mathrm{~Hz}$ & 0.9468 & 0.9175 & 0.7736 & 0.9555 & 0.9240 & 0.8047 & 0.9409 & 0.8849 & 0.7574 & 0.9649 & 0.6458 & 0.7059 \\
\hline $60 \mathrm{~Hz}$ & 0.8961 & 0.8514 & 0.7214 & 0.9013 & 0.8545 & 0.7391 & 0.8882 & 0.8203 & 0.6986 & 0.9141 & 0.5388 & 0.5563 \\
\hline $40 \mathrm{~Hz}$ & 0.8593 & 0.8014 & 0.6737 & 0.8432 & 0.7974 & 0.6885 & 0.8414 & 0.7590 & 0.6478 & 0.8300 & 0.5778 & 0.4977 \\
\hline $20 \mathrm{~Hz}$ & 0.7793 & 0.7050 & 0.5574 & 0.7680 & 0.6735 & 0.5492 & 0.7545 & 0.6752 & 0.5374 & 0.6031 & 0.5577 & 0.3950 \\
\hline
\end{tabular}

[5] S. Erik and E. Kevin, "Electromyogram pattern recognition for control of powered upper-limb prostheses: State of the art and challenges for clinical use," Journal of Rehabilitation Research \& Development, vol. 48, no. 6, pp. 643-659, 2011.

[6] T. Nef and R. Riener, "Armin-design of a novel arm rehabilitation robot," International Conference on Rehabilitation Robotics, pp. 57-60, 2005.

[7] M. Mihelj, T. Nef, and R. Riener, "Armin ii-7 dof rehabilitation robot: mechanics and kinematics," IEEE International Conference on Robotics and Automation, pp. 4120-4125, 2007.

[8] A. Toth, G. Fazekas, G. Arz, and M. Jurak, "Passive robotic movement therapy of the spastic hemiparetic arm with reharob: report of the first clinical test and the follow-up system improvement," International Conference on Rehabilitation Robotics, pp. 127-130, 2005.

[9] D. Yang, "Recognition of hand grasp preshaping patterns applied to prosthetic hand electromyography control," Journal of Mechanical Engineering, vol. 48, no. 15, p. 1, 2012.

[10] Z. Z. Luo and G. Y. Yang, "Prosthetic hand fuzzy control based on touch and myoelectric signal," Robot, vol. 28, no. 2, pp. 224-228, 2006.

[11] J. U. Chu, I. Moon, Y. J. Lee, S. K. Kim, and M. S. Mun, "A supervised feature-projectionbased real-time emg pattern recognition for multifunction myoelectric hand control," IEEE/ASME Transactions on Mechatronics, vol. 12, no. 3, pp. 282-290, 2007.

[12] A. H. Al-Timemy, G. Bugmann, J. Escudero, and N. Outram, "Classification of finger movements for the dexterous hand prosthesis control with surface electromyography," IEEE Journal of Biomedical \& Health Informatics, vol. 17, no. 3, p. 608, 2013.

[13] E. E. Cavallaro, J. Rosen, J. C. Perry, and S. Burns, "Real-time myoprocessors for a neural controlled powered exoskeleton arm," IEEE Transactions on Biomedical Engineering, vol. 53, no. 11, pp. 2387-96, 2006.

[14] F. Zhang, P. Li, Z. G. Hou, Z. Lu, Y. Chen, Q. Li, and M. Tan, "semgbased continuous estimation of joint angles of human legs by using bp neural network," Neurocomputing, vol. 78, no. 1, pp. 139-148, 2012.

[15] Y. Fang, D. Zhou, K. Li, and H. Liu, "Interface prostheses with classifierfeedback based user training," IEEE Transactions on Biomedical Engineering, vol. PP, no. 99, pp. 1-1, 2016.

[16] Y. Sankai, "Hal: Hybrid assistive limb based on cybernics," Springer Tracts in Advanced Robotics, vol. 66, pp. 25-34, 2010.

[17] C. Fleischer and G. Hommel, "A human-exoskeleton interface utilizing electromyography," IEEE Transactions on Robotics, vol. 24, no. 4, pp. 872-882, 2008.

[18] H. Daley, K. Englehart, L. Hargrove, and U. Kuruganti, "High density electromyography data of normally limbed and transradial amputee subjects for multifunction prosthetic control," Journal of Electromyography \& Kinesiology, vol. 22, no. 3, p. 478, 2012.

[19] E. A. Clancy, E. L. Morin, and R. Merletti, "Sampling, noise-reduction and amplitude estimation issues in surface electromyography," Journal of Electromyography and Kinesiology, vol. 12, no. 1, pp. 1-16, 2002.

[20] D. A. Winter, "Biomechanics and motor control of human movement," The Quarterly Review of Biology, 1991.

[21] H. Hermens, B. Freriks, and R. Merletti, "European recommendations for surface electromyography," European recommendations for surface electromyography, 1999.
[22] A. J. Gitter and W. C. Stolov, "Aaem minimonograph \#16: Instrumentation and measurement in electrodiagnostic medicine-part ii," Muscle \& Nerve, vol. 18, no. 8, pp. 799-811, 1995.

[23] S. A. Jø rgensen and A. Fuglsang-Frederiksen, "Turns-amplitude analysis at different sampling frequencies," Electroencephalography \& Clinical Neurophysiology, vol. 81, no. 1, pp. 1-7, 1991.

[24] J. Nilsson, M. Panizza, and M. Hallett, "Principles of digital sampling of a physiologic signal," Electroencephalography \& Clinical Neurophysiology/evoked Potentials, vol. 89, no. 5, p. 349, 1993.

[25] J. C. Ives and J. K. Wigglesworth, "Sampling rate effects on surface emg timing and amplitude measures," Clinical Biomechanics, vol. 18, no. 6 , p. $543,2003$.

[26] Y. Fang, X. Zhu, and H. Liu, "Development of a surface emg acquisition system with novel electrodes configuration and signal representation," Lecture Notes in Computer Science, vol. 8102, pp. 405-414, 2013.

[27] Y. Fang, H. Liu, G. Li, and X. Zhu, "A multichannel surface emg system for hand motion recognition," International Journal of Humanoid Robotics, vol. 12, no. 02, p. 1550011, 2015.

[28] Y. Fang and H. Liu, "Robust semg electrodes configuration for pattern recognition based prosthesis control," IEEE International Conference on Systems, Man and Cybernetics, pp. 2210-2215, 2014.

[29] J. Rafiee, M. A. Rafiee, F. Yavari, and M. P. Schoen, "Feature extraction of forearm emg signals for prosthetics," Expert Systems with Applications, vol. 38, no. 4, pp. 4058-4067, 2011.

[30] K. Englehart and B. Hudgins, "A robust, real-time control scheme for multifunction myoelectric control," IEEE Transactions on Biomedical Engineering, vol. 50, no. 7, p. 848, 2003.

[31] A. Phinyomark, F. Quaine, S. Charbonnier, C. Serviere, F. TarpinBernard, and Y. Laurillau, "Emg feature evaluation for improving myoelectric pattern recognition robustness," Expert Systems with Applications, vol. 40, no. 12, pp. 4832-4840, 2013.

[32] B. Hudgins, P. Parker, and R. N. Scott, "A new strategy for multifunction myoelectric control," IEEE Transactions on Biomedical Engineering, vol. 40, no. 1, pp. 82-94, 1993.

[33] J. Rafiee, M. A. Rafiee, F. Yavari, and M. P. Schoen, "Feature extraction of forearm emg signals for prosthetics," Expert Systems with Applications, vol. 38, no. 4, pp. 4058-4067, 2011. 